\title{
Die Emanzipation der schweigenden Mehrheit
}

\section{Einleitung}

Ob wir nun spezifisch den Umgang des Menschen mit den Wäldern oder seinen Umgang mit der Umwelt generell untersuchen, immer wieder stoßen wir auf soziale Dilemmasituationen. Durch diese Dilemmata werden sehr viele Menschen in ihrem umweltverantwortlichen Handeln gebremst. Warum sollten wir zum Schutze des Waldes auf das Autofahren verzichten, wenn «die anderen» das nicht auch tun? Man fühlt sich alleine einer übergroßen Mehrheit gegenüber. Auch im Rahmen einer am Geographischen Institut der ETH Zürich durchgeführten Studie zur Einstellung der Bevölkerung zu Umwelt und umweltverantwortlichem Handeln in der Schweiz sind wir regelmäßig auf solche Situationsbeschreibungen gestoßen (vgl. REICHERT und ZIERHOFER 1993).

In diesem Beitrag möchte ich der Bedeutung solcher sozialen Dilemmata anhand einer statistischen Auswertung von Daten aus der genannten Untersuchung empirisch nachgehen. Es stellt sich dabei heraus, daß soziale Dilemmata vielfach mehr Einbildung als soziale Wirklichkeit sind. Dies verweist auch auf die Dringlichkeit einer kommunikativen Lösung für diese Dilemmasituationen; sie könnte zu einer handlungsbezogenen «Emanzipation» der Betroffenen führen.

\section{Soziale Dilemmata}

Dilemmata sind Ausdruck des Scheiterns einer bestimmten Rationalität. Als solche sind sie auch in der Ökonomie, die in ihrer Hauptströmung weitgehend individualistische Rationalitätsannahmen trifft, bekannt und vielfach erforscht (vgl. z.B. WEIMANN 1991). Sie treten in Situationen auf, die die theoretische Annahme, daß auch kollektive Interessen - wie z. B. die Erhaltung des Waldes - am effizientesten durch das konsequente Verfolgen individueller Nutzenziele befriedigt werden können, in Frage stellen. Soziale Dilemmata bringen das fundamentale Problem der Übereinstimmung von individueller und kollektiver Rationalität an die Oberfläche. Anhand dieser sozialen Dilemmata treten uns die heutigen Umweltprobleme als Koordinations- und Kooperationsprobleme entgegen. Verschiedentlich ist versucht worden, diese sozialen Dilemmata spieltheoretisch zu formulieren und zu analysieren.
Das klassische Beispiel der spieltheoretischen Formulierung eines sozialen Dilemmata ist das sogenannte Gefangenendilemma. Die oben erwähnten sozialen Dilemmasituationen' sind damit vergleichbar. So beschreiben sie Situationen, in denen ein Individuum einen Beitrag zur Verminderung der Umweltschädigung liefert (z. B. indem er oder sie weniger Auto fährt), vom Nutzen eines solchen Beitrages (etwa in Form eines Waldspazierganges in einem gesunden Wald oder des Schutzes des Bannwaldes) jedoch nicht allein profitieren kann. Verhandlungen oder sonstige kommunikative Einigungen über einen Interessen- oder Kostenausgleich sind aus irgendwelchen Gründen, z. B. wegen des Fehlens institutionalisierter Kommunikationskanäle oder sonstiger zu diesem Zweck eingerichteter demokratischer Institutionen, ausgeschlossen. Als Konsequenz ist der individuelle Nutzen des weiteren Gebrauchs des Autos relativ groß im Vergleich zum individuellen Nutzen, den eine saubere Luft mit sich bringt. Unter der Voraussetzung individuell rationalen Verhaltens führt dies zu einer Situation, in der keine umweltverantwortlichen Handlungen zustande kommen, in der also nicht auf das Auto verzichtet wird. Das Resultat individuell rationalen Verhaltens ist kollektiv gesehen nichtrational. Eine der wichtigsten Fragen der Umweltforschung ist deshalb die nach der Lösbarkeit solcher Dilemmasituationen. Wie kann es zu kooperativem, umweltverantwortlichem Handeln kommen?

Es ist von verschiedener Seite behauptet worden, daß es die individuelle Neigung zu Kooperation oder Altruismus ist, die bestimmt, inwieweit ein Mensch überhaupt kooperiert bzw. umweltverantwortlich handelt. Diese These hat sich aber empirisch nicht hinreichend bestätigen lassen (vgl. dazu unter anderen SEN 1977 und SUDGEN 1984). Eine interessante Alternative, die auch ich hier aufgreifen möchte, ist die Vorstellung, daß ein enges Konzept von Rationalität nicht haltbar ist, daß das menschliche umweltverantwortliche Handeln auch durch Normen gesteuert wird. Der Begriff der Rationalität ist dann viel weiter zu fassen. Genau dies hat Jürgen

Huib Ernste, Dr., Wirtschafts- und Sozialgeograph, Geographisches Institut der ETH, Winterthurerstr. 190, 8057 Zürich 
HABERMAS in seiner Theorie des Kommunikativen Handelns (1987) versucht, und ich möchte seinen Gedanken hier anhand der empirischen Befunde ansatzweise weiterführen.

HABERMAS zeigt, daß die herkömmliche Auffassung von Rationalität als rein zweckrationales Handeln oder übersetzt in die Terminologie der utilitaristischen Ökonomie - als individuellen Nutzen maximierendes Handeln der sozialen Realität nicht gerecht werden kann. Diese enge Form des Rationalitätsbegriffes ist bereits im Werk von Max WEBER anzutreffen. HABERMAS hält zwar an ihr fest, erweitert diese jedoch um moralischpraktische und ästhetisch-expressive Momente (REESESCHÄFER 1991, S. 34). Alle Komponenten zusammen machen das aus, was HABERMAS als kommunikative Rationalität bezeichnet. Diese hat sich im Zuge der kulturellen Evolution aus einer vorsprachlichen Intersubjektivität der Menschen entwickelt, und jeder Mensch wächst durch den Sozialisationsprozeß in sie hinein. In dieser auf kulturell geprägten Sprachstrukturen aufbauenden kommunikativen Rationalität sind solidarisierende Momente eingeschlossen (KUNNEMAN 1985). ${ }^{2}$ Unter diesen Voraussetzungen sollte eine effektive Umweltpolitik nicht so sehr auf die Schaffung von Rahmenbedingungen zum Einbezug der Umwelt in ein utilitaristisches Handlungskalkül ausgerichtet sein, sondern der möglichst ungehinderten Entfaltung einer kommunikativen Rationalität förderlich sein. Aus schweigenden, in Dilemmasituationen gefangenen Individuen sollten kommunikativ orientierte, emanzipierte und umweltverantwortlich handelnde Mitglieder unserer Gesellschaft werden. Diese Kommunikation ist es auch, die unter dem Schlagwort «Risikodialog» heute von verschiedenen Seiten gefordert wird und von der man sich die Auflösung der Dilemmasituationen verspricht. Für die Wissenschaft läßt sich daraus die Suche nach einem Programm ableiten, das nach den Bedingungen für die Entwicklung einer Art von kommunikativem Handeln fragt, das den Aufbau von gegenseitigem Verständnis, Vertrauen und Kooperationswillen fördern kann. Die nachfolgenden empirischen Ergebnisse sollen dazu einen ersten Beitrag leisten.

\section{Erste Vermutungen und empirische Ergebnisse}

In einem ersten Schritt dieser statistischen Nachanalyse der im Rahmen des Forschungsprojektes «Umwelt zur Sprache bringen» erhobenen Daten zu umweltrelevanten Einstellungen und Handlungen der Schweizer Bevölkerung $^{3}$ werde ich versuchen, die Dilemmasituation, in der sich viele Leute wähnen, aufzuzeigen. Im Sinne des oben erwähnten Forschungsprogramms drängt sich dann die Frage auf, ob sich aus den übrigen Daten zu diesen Personen Hinweise für eine auf kommunikative Rationalität aufbauende Umweltpolitik ableiten lassen. Anlaß zu dieser Analyse waren die Antworten auf die folgende, von uns im Fragebogen gestellte Frage:

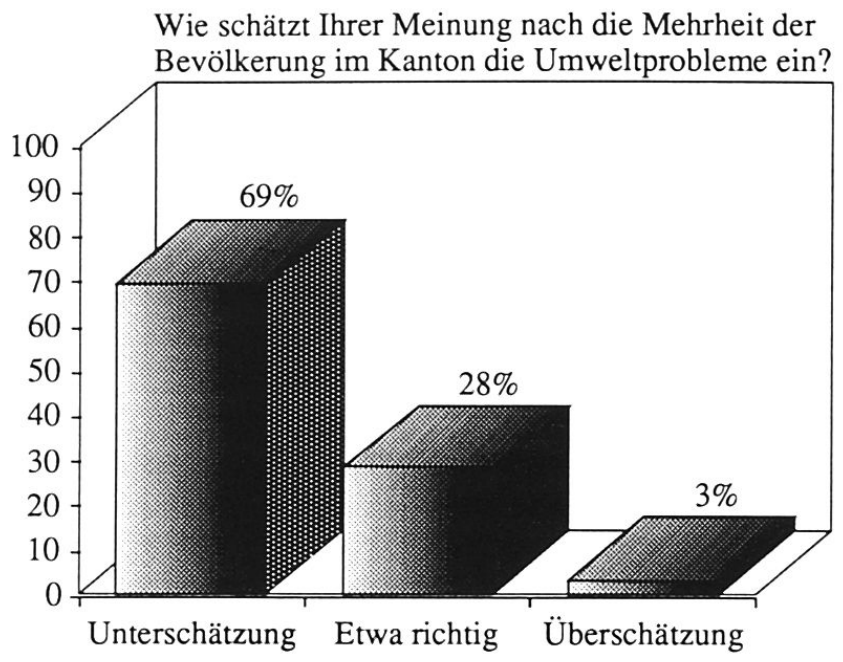

Figur 1 Geschätzte Meinung der Kantonsmehrheit.

Figur 1 gibt das Ergebnis einer gewichteten Hochrechnung für den ganzen Kanton Tessin wieder. Bei den Befragten ist die Meinung, daß die Kantonsmehrheit die Umweltprobleme unterschätzt, sehr weit verbreitet (69\%), womit der Kantonsmehrheit ein deutlicher Mangel an Umweltbewußtsein attestiert wird. Dabei ist das, was die Befragten als eine Unterschätzung bezeichnen, selbstverständlich davon abhängig, was sie selbst als eine richtige Einschätzung erachten. Die Meinung, daß die Kantonsmehrheit die Umweltprobleme unterschätzt, deutet also auch darauf hin, daß die Befragten selbst das Gefühl haben, die Umweltprobleme ernster zu nehmen als jene Mehrheit. Fast 70 Prozent der Bevölkerung zählen sich selbst nicht zu dieser Kantonsmehrheit von umwelt unbewußteren Leuten, und hierin liegt nun gerade die Brisanz dieses Ergebnisses. Die Mehrheit der Bevölkerung glaubt sich in der Minderheit. Die Mehrheit vermutet, daß "die anderen" die Umweltprobleme anders einschätzen, und zwar so, daß sie für umweltverantwortlicheres Handeln nicht zu gewinnen sind und lieber eine Trittbrettfahrer-Option wählen. Es besteht also ein unberechtigtes Mißtrauen in die (kognitive) Fähigkeit der Kantonsmehrheit zur richtigen Einschätzung der Umweltprobleme. Daß sich die Mehrheit in einer Minderheit wähnt, läßt auch die Vermutung zu, daß das selbst deklarierte Umweltbewußtsein der "anderen» oft nicht als Umweltbewußtsein erkannt oder anerkannt wird. Es stehen sich offenbar verschiedene Konzeptionen von Umweltbewußtsein bzw. einer richtigen Einschätzung der Umweltprobleme gegenüber.

Die Schlußfolgerung auf Grund dieser einen Frage, nämlich daß die Tessiner Kantonsmehrheit sich in einer Dilemmasituation befindet, die ein umweltverantwortliches Handeln bremst oder hindert, würde allerdings die Aussagekraft der Statistik allzusehr strapazieren. Im nachfolgenden Abschnitt wollen wir der Frage, wie viele und welche Leute tatsächlich ein Gefühl von Ohnmacht 
oder von Sinnlosigkeit in bezug auf umweltverantwortliches Handeln in Dilemmasituationen haben, empirisch näher nachgehen.

\section{Die Ermittlung des Ohnmachtsgefühls}

Für eine breiter abgestützte Ermittlung des Ohnmachtsgefühls der Befragten lassen sich eine Reihe weiterer Fragen unseres Fragebogens beiziehen. Tabelle 1 gibt die für den ganzen Kanton hochgerechneten Resultate wieder:
Tabelle 1 Hochgerechnete Antworthäufigkeit bei den weiteren Fragen zur Ohnmachtsempfindung.

\begin{tabular}{|c|c|c|c|}
\hline $\begin{array}{l}\text { Welche der folgenden } \\
\text { Aussagen treffen } \\
\text { auf Sie zu? }\end{array}$ & $\mathrm{Ja}$ & Nein & $\begin{array}{l}\text { kann ich } \\
\text { nicht } \\
\text { sagen }\end{array}$ \\
\hline $\begin{array}{l}\text { Manchmal zweifle ich, ob } \\
\text { ich als Einzelperson etwas } \\
\text { zur Verbesserung der } \\
\text { Umweltsituation beitragen } \\
\text { kann }\end{array}$ & $66 \%$ & $34 \%$ & \\
\hline $\begin{array}{l}\text { Es kommt vor, dass ich } \\
\text { mich ohnmächtig fühle }\end{array}$ & $88 \%$ & 9\% & $3 \%$ \\
\hline
\end{tabular}

Flugplatzgebühren nach Menge der Abgase für jedes Flugzeug

Informationskampagne über die Probleme der Luftverschmutzung

Weitere Förderung des kombinierten Verkehrs Strasse-Schiene für den Schwerverkehr

Forschungsprogramm über die Ursachen und Folgen des Ozons in der Luft

Finanzhilfe zur freiwilligen Reduktion des Schadstoffausstosses in der Industrie

Benzinpreis erhöhen

Velowege, Fussgängerzonen und Bussfahrbahnen in den Stadtzentren und Stauzonen

Begrenzung der Anzahl öffentlicher Parkplätze in den Stadtzentren

Weiterer Ausbau des öffentlichen Verkehrs zwischen den grösseren Ortschaften

Steuer auf Lösungsmittel und Lackfarben, die in der Industrie verwendet werden, bei denen Gase entweichen

Geschwindigkeitsreduktion: $100 \mathrm{~km} / \mathrm{h}$ auf Autobahnen, $80 \mathrm{~km} / \mathrm{h}$ auf Landstrassen

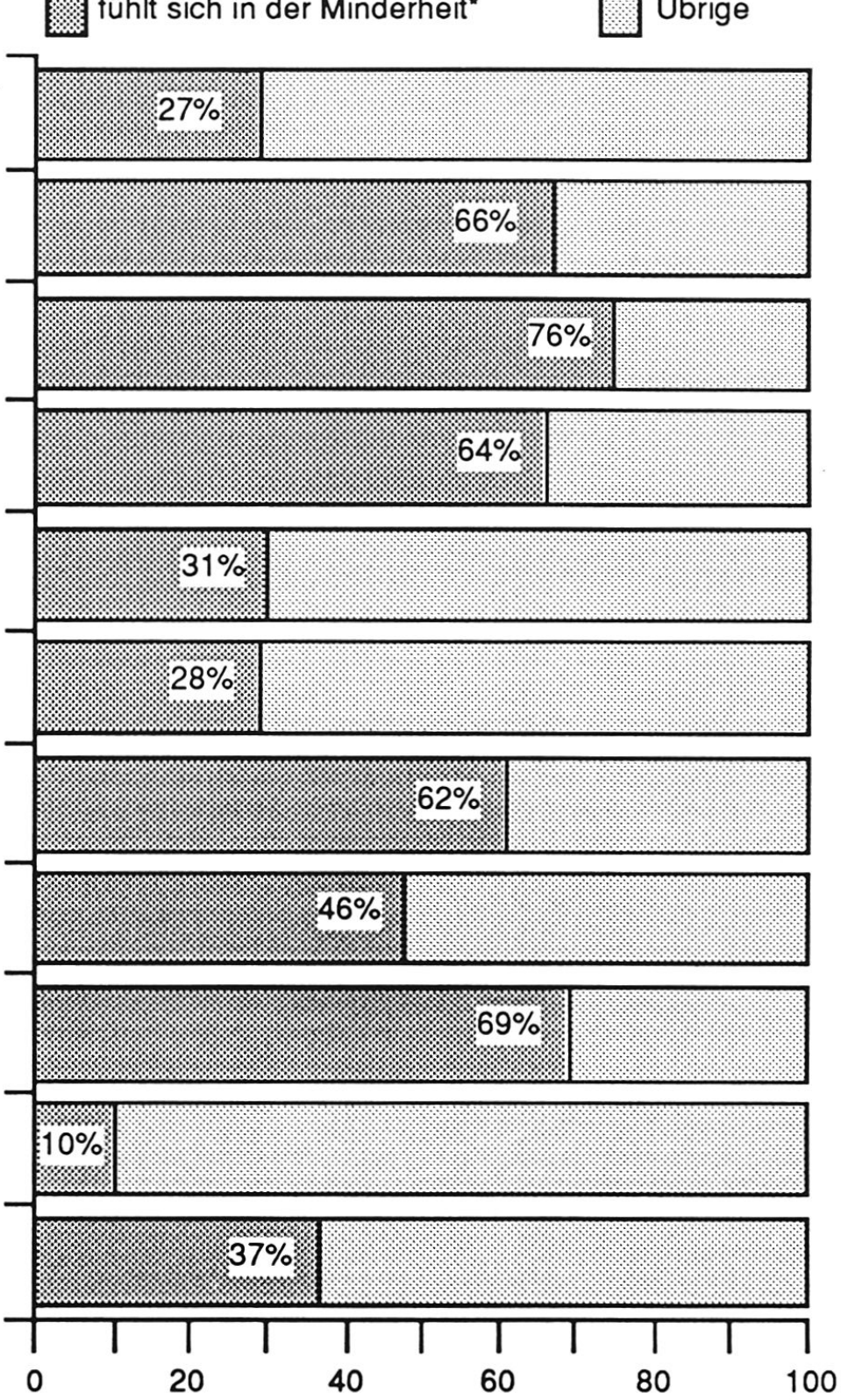

"Anteil der Leute, die meinen, dass die Kantonsmehrheit die aufgelisteten Massnahmen als weniger dringlich einstuft, als sie dies selbst tun

Figur 2 Subjektiv empfundene Minderheitssituation bei den Fragen zu Luftreinhaltemaßnahmen im Kanton Tessin. 
Unsere erste Vermutung, daß sich ein übergroßer Anteil der Bevölkerung einer weniger umweltbewußten Mehrheit gegenüber sieht und sich aus diesem Grund in ihrem umweltverantwortlichen Handeln gebremst glaubt, scheint sich durch das Ergebnis dieser Fragen zu bestätigen. Eine erdrückende Mehrheit der Bevölkerung bringt zum Ausdruck, daß sie sich nicht mächtig genug fühlt, sich hinreichend für die Umwelt einzusetzen.

Verschiedene Fragen zu spezifischen Maßnahmen der Luftreinhaltung zeigen ein ähnliches Bild. ${ }^{4}$ Es wurde einerseits nach der persönlichen Meinung zu diesen einzelnen Maßnahmen gefragt, anderseits aber auch nach der geschätzten Meinung der Kantonsmehrheit zu diesen gleichen Maßnahmen. Wenn nun die Befragten eine Maßnahme als dringlicher einstufen, als dies nach ihrer Meinung die Kantonsmehrheit tut, so ist dies wiederum ein Hinweis darauf, daß sie sich mit ihrer Meinung in einer Minderheit wähnen. Figur 2 gibt für jede der fraglichen Luftreinhaltemaßnahmen die Zahl jener Leute an, die in diesem Sinne eine Einstellung zu haben glauben, die umweltverantwortlicher ist als die der Kantonsmehrheit, die sich also subjektiv in einer Minderheit wähnen. Wir können uns die Frage stellen, wie weit solche Merkmale als Indikatoren für das subjektiv empfundene Minderheitsgefühl brauchbar sind.

Aus den Antworten auf diese Frage läßt sich anhand der Hochrechnung aber auch ableiten, wie die Situation objektiv aussieht, d. h., was die Kantonsmehrheit tatsächlich meint (vgl. Figur 3). Für jeden Befragten kann durch den Vergleich seiner persönlichen Antwort mit jener, die für die Kantonsmehrheit ermittelt wurde, festgestellt werden, ob er oder sie sich zu Recht oder zu Unrecht in

dagegen weniger dringlich sehr dringlich

Flugplatzgebühren nach Menge der Abgase für jedes Flugzeug

Informationskampagne über die Probleme der Luftverschmutzung

Weitere Förderung des kombinierten Verkehrs Strasse-Schiene für den Schwerverkehr

Forschungsprogramm über die Ursachen und Folgen des Ozons in der Luft

Finanzhilfe zur freiwilligen Reduktion des Schadstoffausstosses in der Industrie

Benzinpreis erhöhen

Velowege, Fussgängerzonen und Bussfahrbahnen in den Stadtzentren und Stauzonen

\section{Begrenzung der Anzahl öffentlicher Parkplätze} in den Stadtzentren

\section{Weiterer Ausbau des öffentlichen Verkehrs zwischen den grösseren Ortschaften}

Stever auf Lösungsmittel und Lackfarben, die in der Industrie verwendet werden, bei denen Gase entweichen

Geschwindigkeitsreduktion: $100 \mathrm{~km} / \mathrm{h}$ auf Autobahnen, $80 \mathrm{~km} / \mathrm{h}$ auf Landstrassen

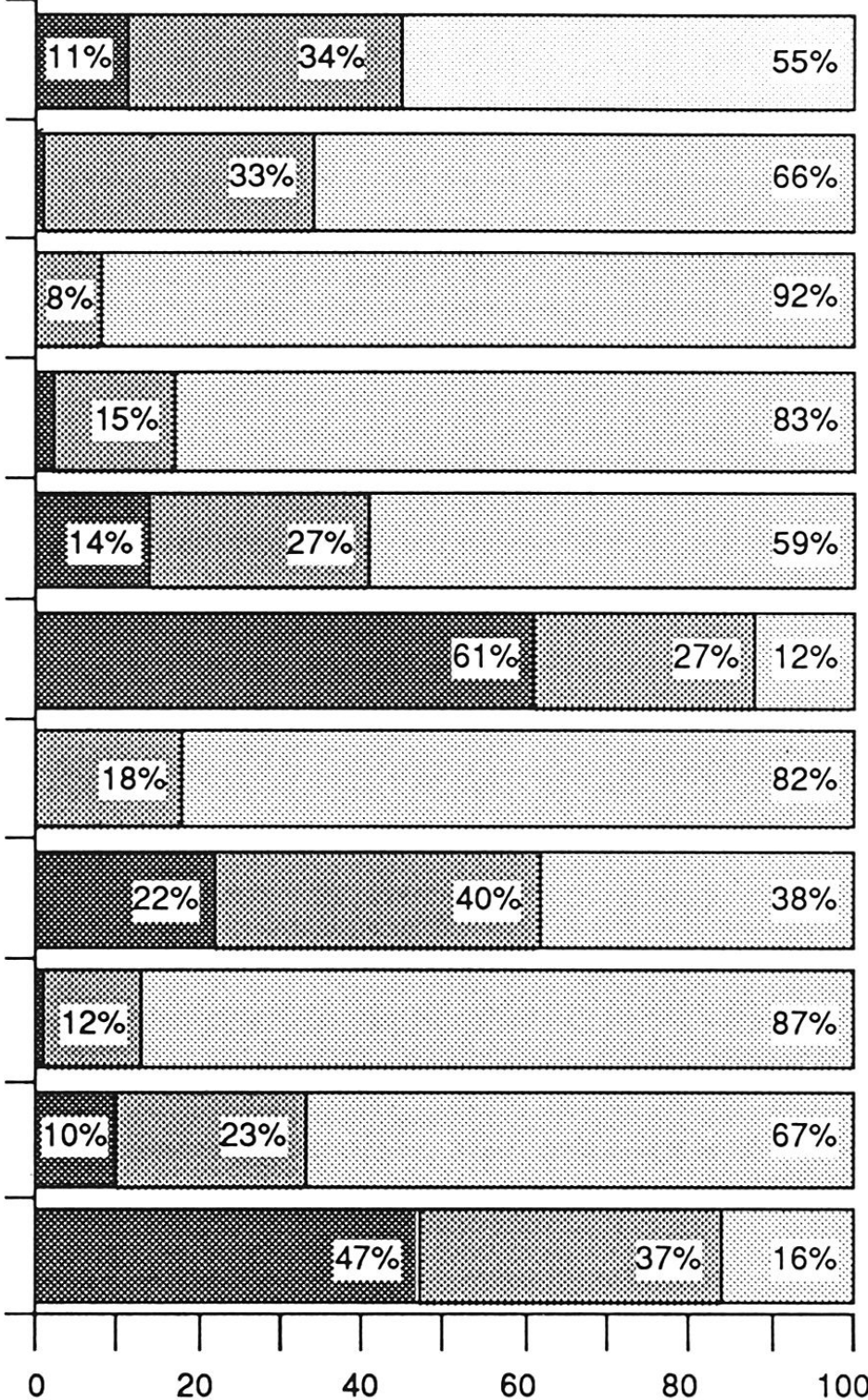

Figur 3 Hochrechnung der persönlichen Dringlichkeitseinschätzungen für die Luftreinhaltemaßnahmen im Kanton Tessin. 
der Minderheit wähnt. ${ }^{5}$ Es zeigt sich, daß dies in den meisten Fällen zu Unrecht geschieht. Nur bei der Stellungnahme hinsichtlich einer Benzinpreiserhöhung war die Einschätzung, daß man sich in einer Minderheitsposition befinde, zu 92\% richtig. Bei der Frage nach der Geschwindigkeitsreduktion betrug dieser Prozentsatz dagegen nur 33\% und bei der nach der Begrenzung der Anzahl öffentlicher Parkplätze lediglich 24\%. In allen übrigen Fällen war die Vermutung, einer Minderheit anzugehören, zu $100 \%$ falsch!

Das «generalisierte Minderheits-/Ohnmachtsgefühl»

Die Antworten auf die bisher betrachteten Fragen weisen untereinander deutliche Zusammenhänge auf. Es darf deshalb erwartet werden, daß sie auch als Indikatoren zur Beschreibung eines generalisierten Minderheits-/
Ohnmachtsgefühls dienen könnten. Gelingt es, ein derartiges Maß zu entwickeln, werden damit generellere Aussagen über den Zusammenhang des Minderheits-/Ohnmachtsempfindens mit den übrigen uns in diesem Kontext interessierenden Merkmalen aus unserem Datensatz möglich, und es darf die Hoffnung genährt werden, daraus Hinweise zur Überwindung dieses Empfindens gewinnen zu können.

Um genau diese Möglichkeiten abzutasten, wurde eine zweistufige sogenannte Latente-Klassen-Analyse durchgeführt. ${ }^{6}$ In einem ersten Schritt fanden die Stellungnahmen zu den Luftreinhaltemaßnahmen Verwendung, um zu einer Einstufung der Befragten auf einer (Nominal-) Skala des Minderheitsgefühls bzw. zu ihrer Einordnung in verschiedene (latente) Klassen ${ }^{7}$ zu gelangen, die sich nach dem empfundenen Minderheitsgefühl unterscheiden. Es zeigte sich, daß sinnvollerweise zwei solche Klassen unterschieden werden können: Tabelle 2 gibt ihre

Tabelle 2 Klassenprofile bezüglich des Minderheitsgefühls bei den Meinungen zu den Luftreinhaltemaßnahmen.

\begin{tabular}{|c|c|c|c|}
\hline (Manifeste) Variablen & $\begin{array}{l}\text { Katego- } \\
\text { rien* }\end{array}$ & $\begin{array}{c}\text { Bedingt } \\
\text { scheinl } \\
1\end{array}$ & $\begin{array}{l}- \\
\text { en } \\
2\end{array}$ \\
\hline $\begin{array}{l}\text { Geschwindigkeitsreduktion: } 100 \mathrm{~km} / \mathrm{h} \text { auf Autobahnen, } \\
80 \mathrm{~km} / \mathrm{h} \text { auf Landstrassen }\end{array}$ & $\begin{array}{l}1 \\
2\end{array}$ & $\begin{array}{l}0,4 \\
0,6\end{array}$ & $\begin{array}{l}0,8 \\
0,2\end{array}$ \\
\hline $\begin{array}{l}\text { Steuer auf Lösungsmittel und Lackfarben, die in der } \\
\text { Industrie verwendet werden, bei denen Gase entweichen }\end{array}$ & $\begin{array}{l}1 \\
2\end{array}$ & $\begin{array}{l}0,9 \\
0,1\end{array}$ & $\begin{array}{l}0,9 \\
0,1\end{array}$ \\
\hline $\begin{array}{l}\text { Weiterer Ausbau des öffentlichen Verkehrs zwischen den } \\
\text { grösseren Ortschaften }\end{array}$ & $\begin{array}{l}1 \\
2\end{array}$ & $\begin{array}{l}0,6 \\
0,4\end{array}$ & $\begin{array}{l}0,1 \\
0,9\end{array}$ \\
\hline $\begin{array}{l}\text { Begrenzung der Anzahl öffentlicher Parkplätze in den } \\
\text { Stadtzentren }\end{array}$ & $\begin{array}{l}1 \\
2\end{array}$ & $\begin{array}{l}0,9 \\
0,1\end{array}$ & $\begin{array}{l}0,3 \\
0,7\end{array}$ \\
\hline $\begin{array}{l}\text { Velowege, Fusgängerzonen und Busfahrbahnen in den } \\
\text { Stadtzentren und Stauzonen }\end{array}$ & $\begin{array}{l}1 \\
2\end{array}$ & $\begin{array}{l}0,7 \\
0,3\end{array}$ & $\begin{array}{l}0,2 \\
0,8\end{array}$ \\
\hline Benzinpreis erhöhen & $\begin{array}{l}1 \\
2\end{array}$ & $\begin{array}{l}0,6 \\
0,4\end{array}$ & $\begin{array}{l}0,8 \\
0,2\end{array}$ \\
\hline $\begin{array}{l}\text { Finanzhilfe zur freiwilligen Reduktion des } \\
\text { Schadstoffausstosses in der Industrie }\end{array}$ & $\begin{array}{l}1 \\
2\end{array}$ & $\begin{array}{l}0,5 \\
0,5\end{array}$ & $\begin{array}{l}0,8 \\
0,2\end{array}$ \\
\hline $\begin{array}{l}\text { Forschungsprogramm über die Ursachen und Folgen des } \\
\text { Ozons in der Luft }\end{array}$ & $\begin{array}{l}1 \\
2\end{array}$ & $\begin{array}{l}0,6 \\
0,4\end{array}$ & $\begin{array}{l}0,2 \\
0,8\end{array}$ \\
\hline $\begin{array}{l}\text { Weitere Förderung des kombinierten Verkehrs Strasse- } \\
\text { Schiene für den Schwerverkehr }\end{array}$ & $\begin{array}{l}1 \\
2\end{array}$ & $\begin{array}{l}0,5 \\
0,5\end{array}$ & $\begin{array}{l}0,1 \\
0,9\end{array}$ \\
\hline $\begin{array}{l}\text { Informationskampagne über die Probleme der } \\
\text { Luftverschmutzung }\end{array}$ & $\begin{array}{l}1 \\
2\end{array}$ & $\begin{array}{l}0,6 \\
0,4\end{array}$ & $\begin{array}{l}0,2 \\
0,8\end{array}$ \\
\hline $\begin{array}{l}\text { Flugplatzgebühren nach Menge der Abgase für jedes } \\
\text { Flugzeug }\end{array}$ & $\begin{array}{l}1 \\
2\end{array}$ & $\begin{array}{l}0,5 \\
0,5\end{array}$ & $\begin{array}{l}0,9 \\
0,1\end{array}$ \\
\hline \multicolumn{2}{|l|}{ Latente Klassen-Wahrscheinlichkeit } & 0,4 & 0,6 \\
\hline
\end{tabular}

* 1 = Die befragte Person vermutet, dass sie die betreffende Massnahme nicht als dringlicher einstuft als dies die Kantonsmehrheit tut. 2 = Die befragte Person vermutet, dass sie die betreffende Massnahme als dringlicher einstuft als dies die Kantonsmehrheit tut 
Profile wieder. ${ }^{8}$ Die bedingten Wahrscheinlichkeiten drücken die relativen Häufigkeiten aus, mit denen die einzelnen Kategorien innerhalb der betreffenden Klasse vertreten sind. Die Latente-Klassen-Wahrscheinlichkeiten beziehen sich auf die relative Größe dieser Klassen. Ein Blick auf Tabelle 2 zeigt uns, daß die zweite latente Klasse mehrheitlich jene Leute repräsentiert, die sich in bezug auf eine Reihe von Luftreinhaltemaßnahmen eher in einer Minderheitsposition wähnen. Bei 6 von 11 Luftreinhaltemaßnahmen ist für eine befragte Person die Wahrscheinlichkeit der Zugehörigkeit zur Kategorie 2 (es wird vermutet, daß man selbst die Dringlichkeit einer Maßnahme höher einschätzt, als die Kantonsmehrheit dies tut) größer als die der Zugehörigkeit zur Kategorie 1. In der ersten latenten Klasse liegt das Verhältnis umgekehrt. Bei 7 von 11 Maßnahmen ist die Wahrscheinlichkeit der Zugehörigkeit zur Kategorie 1 (es wird vermutet, daß man selbst die Dringlichkeit einer Maßnahme nicht höher einschätzt, als die Kantonsmehrheit dies tut) gröBer als die der Zugehörigkeit zur Kategorie 2. Bei den übrigbleibenden 4 Maßnahmen gibt es in 3 Fällen eine Gleichverteilung bezüglich der Kategorien 1 und 2. Weiter sehen wir wiederum, daß die Gruppe der Leute, die sich «ohnmächtig» fühlen, in der Mehrzahl ist, denn die erste latente Klasse umfaßt ungefähr 40, die zweite ungefähr $60 \%$ der Gesamtbevölkerung. Unsere ersten Vermutungen haben sich somit bereits weiter verfestigt. ${ }^{9}$

In einem zweiten Schritt wurde dann diese Skala (bzw. diese Einteilung in zwei latente Klassen) für das Minderheitsgefühl bezüglich der Luftreinhaltemaßnahmen mit den drei in Figur 1 und Tabelle 1 erwähnten Fragen kombiniert, um die Befragten auf einer endgültigen Skala für das generalisierte Ohnmachts-/Minderheitsgefühl einstufen zu können. ${ }^{10}$ Das Resultat ist in Tabelle 3 dargestellt. Es zeigt, daß sich unsere Grundgesamtheit bezüglich des Ohnmachts-/Minderheitsgefühls in drei Klassen einteilen läßt, wobei die erste Klasse am deutlichsten jene Bevölkerungsgruppe repräsentiert, die sich im $\mathrm{Zu}$ sammenhang der hier betrachteten Aspekte in der Minderheit wähnt. Mit Ausnahme der Zuordnung bei den Luftreinhaltemaßnahmen (Gleichverteilung) tendieren die Mitglieder dieser Gruppe eindeutig zu jener Antwortkategorie, die ein Minderheitsgefühl anzeigt. Und wiederum ist diese Klasse auch deutlich in der Mehrheit. Dazu kommt, daß auch die beiden anderen Klassen zum Teil Leute umfassen, die sich in gewisser Hinsicht ohnmächtig fühlen. Dabei ist die zweite Gruppe etwas ambivalent. Einerseits zweifeln ihre Mitglieder nicht daran, daß man etwas zur Verbesserung der Umwelt beitragen kann, andererseits fühlen sie sich in gewissen Bereichen oft ohnmächtig. Die Mitglieder der dritten Klasse fühlen sich in ihrem Urteil über Umweltprobleme zwar nicht so sehr in einer Minderheitssituation, empfinden aber trotzdem auch bezüglich verschiedener Aspekte ein gewisses Ohnmachtsgefühl, wenn auch etwas schwächer, als dies bei der ersten Gruppe der Fall ist.

\section{Schlußfolgerungen}

Der Ausgangspunkt dieser empirischen Analyse war der Versuch, die These, daß ein Großteil der Bevölkerung ungerechtfertigterweise glaubt, in einer Dilemmasituation zu stecken, auf festeren Boden zu stellen. Wir müssen annehmen, daß solche subjektiven Empfindungen viele von uns an umweltverantwortlichem Handeln hindern. Es ist deshalb wichtig, feststellen zu können, daß es tatsächlich viel mehr "Ähnlichgesinnte» gibt. Statt daß wir uns mißtrauisch gegenüberstehen, sollten wir uns überlegen, wie ein gegenseitiger Verständigungsproze $ß$ in Gang gebracht werden könnte.

Auch die Ergebnisse vieler bisheriger spieltheoretischer Experimente zeigen, daß die interpersonelle kommunikative Auseinandersetzung eine überaus entscheidende

Tabelle 3 Klassenprofile für die Skala des generalisierten Minderheits-/Ohnmachtsgefühls.

\begin{tabular}{lcccc}
\hline (Manifeste) Variablen & $\begin{array}{l}\text { Katego- } \\
\text { rien }\end{array}$ & Bedingte & Wahrscheinlichkeiten \\
& & $\mathbf{1}$ & $\mathbf{2}$ & $\mathbf{3}$ \\
\hline Minderheitsgefühl bezüglich der Einschätzung von & 1 & 0,8 & 0,8 & 0,3 \\
Umweltprobleme & 2 & 0,2 & 0,2 & 0,7 \\
& 1 & 1,0 & 0,8 & 0,7 \\
Es kommt vor, dass ich mich ohnmächtig fühleb & 2 & 0,0 & 0,0 & 0,1 \\
Manchmal zweifle ich, ob ich als Einzelperson etwas zur & 3 & 0,0 & 0,2 & 0,2 \\
Verbesserung der Umweltsituation beitragen kannc & 2 & 0,9 & 0,0 & 0,6 \\
Kategorien der latenten Skala für das Minderheitsgefühl & 1 & 0,1 & 1,0 & 0,4 \\
bezüglich der Luftreinhaltemassnahmen (vgl. Tabelle 2) & 2 & 0,5 & 0,8 & 0,0 \\
\hline Latente Klassen-Wahrscheinlichkelt & & 0,5 & 0,2 & 1,0 \\
\hline
\end{tabular}

$a_{1}=$ Fühlt sich in der Minderheit, $2=$ Fühlt sich nicht in der Minderheit. $b_{1}=\mathrm{Ja}, 2=$ Kann ich nicht sagen, $3=$ Nein. $\mathrm{C}_{1}=\mathrm{Ja}, 2=$ Nein 
Rolle bei der Überwindung sozialer Dilemmasituationen spielt (DAWES, McTAVISH und SCHAKLEE 1977, DAWES 1980 und BRECHNER 1977). Dies bezieht sich sowohl auf den Bereich einer mehr allgemeinen kulturellen Auseinandersetzung (humanisation) als auch auf Kommunikation, die konkreter auf umweltrelevantes Handeln gerichtet ist (discussion), und auf formale Verhandlungen und Abmachungen (commitments)." Nur mittels einer solchen kommunikativen Thematisierung und Begründung von Absichten, Erwartungen und kurz- und langfristigen reaktiven Verhaltensweisen kann eine Vertrauensbasis entstehen, die kooperatives, umweltverantwortlicheres Handeln möglich macht. In REICHERT und ZIERHOFER (1993) werden diverse Ansätze dazu ausführlich dargestellt. Die Ergebnisse der vorliegenden kleinen Analyse zu Ausmaß und Verbreitung eines «Ohnmachts»-Gefühls lassen vermuten, daß auf dem Weg der Bekämpfung eines solchen Gefühls deutlich mehr erreicht werden könnte als z. B. mit einer verstärkten Einführung von umweltrelevanten Verboten und Geboten. In ähnlichem Sinne haben auch EDNEY und HARPER bereits 1978 überzeugend dargelegt, daß Kontroll- und Sanktionsmaßnahmen nur dann effektiv sein können, wenn sie durch einen demokratischen, kommunikativ-rationalen Diskurs legitimiert worden sind. Mit andern Worten, nur wenn aus schweigenden, in Dilemmasituationen gefangenen Individuen kommunikativ orientierte, emanzipierte und umweltverantwortlich handelnde Mitglieder unserer Gesellschaft werden, läßt sich der Wald nachhaltig nutzen und erhalten.

\section{Anmerkungen}

Diese umweltbezogenen sozialen Dilemmasituationen werden manchmal auch anhand des von HARDIN (1968) eingeführten Beispiels der Allmendklemme oder der "tragedy of the commons" beschrieben.

${ }^{2}$ Eine detaillierte Darstellung der Theorie von Habermas würde den Rahmen dieses Beitrags sprengen. Für eine Übersicht über ihre Bedeutung für die Umweltforschung vgl. auch REICHERT und ZIERHOFER (1993).

${ }^{3}$ Da die Stichprobe für die standardisierte Befragung hauptsächlich aus der Wohnbevölkerung des Kantons Tessin gezogen wurde, ist eine gewisse Vorsicht bei der Generalisierung auf die ganze Schweiz angebracht.

${ }^{4}$ Die Fragen lauteten: "Es folgt eine Liste mit Vorschlägen zur Luftreinhaltung. Was halten Sie von diesen Maßnahmen? bzw. "Hier folgt noch einmal dieselbe Liste mit Vorschlägen zur Luftreinhaltung. Jetzt möchten wir aber gerne wissen, wie Sie die Meinungen in Ihrem Kanton einschätzen. Was hält Ihrer Meinung nach die Mehrheit der Tessinerinnen und Tessiner von diesen Maßnahmen?"

${ }^{5}$ Ein Beispiel kann dies verdeutlichen: Eine Person ist der Meinung, Geschwindigkeitsreduktionen seien sehr dringlich, glaubt aber, daß die Kantonsmehrheit diese Maßnahme weniger dringlich findet oder sogar gänzlich dagegen eingestellt ist. Aus der Hochrechnung der persönlichen Meinungen ergibt sich nun, daß knapp 37\% der Bevölkerung im Kanton Tessin diese Maßnahme als weniger dringlich einstufen und $47 \%$ sogar ganz dagegen sind. Zusammen betrach- ten also 84\% der Tessiner Bevölkerung Geschwindigkeitsreduktionen als weniger dringlich als die fragliche Person. Sie fühlt sich in diesem Fall somit zu Recht in der Minderheit. Eine andere Person findet Geschwindigkeitsreduktionen etwas weniger dringlich und meint gleichzeitig, daß die Kantonsmehrheit völlig dagegen sei. Auch sie fühlt sich mit ihrer Meinung also in einer Minderheitsposition. Wie schon gesagt, zeigen die Hochrechnungen aber, daß nur $47 \%$ der Kantonsbevölkerung sich völlig gegen Geschwindigkeitsreduktionen aussprechen. Die betreffende Person fühlt sich in diesem Fall also ungerechtfertigterweise in der Minderheit. Eine Mehrheit der Tessiner Bevölkerung, wenn auch eine nicht sehr große, findet Geschwindigkeitsreduktionen mindestens so dringlich wie die fragliche Person.

${ }^{6}$ Die Latente-Klassen-Analyse ist ein statistisches Verfahren, das mit der klassischen Faktorenanalyse vergleichbar ist. Nur werden nicht metrisch skalierte manifeste Merkmale auf metrisch skalierte latente Faktoren, sondern nominal skalierte manifeste Variablen auf eine nominal skalierte latente Variable abgebildet. Für eine eingehendere Darstellung dieser Art der Analyse und ihrer Anwendungsmöglichkeiten verweise ich auf McCUTCHEON (1987), CLOGG und GOODMAN (1984) und HAGENAARS (1990). Für Anwendungen im Bereich der Geographie vgl. ERNSTE und FISCHER (1991).

${ }^{7}$ Man spricht hier im Gegensatz zu den direkt beobachteten (manifesten) Stellungnahmen zu den einzelnen Luftreinhaltemaßnahmen von latenten Klassen, da diese nicht direkt beobachtbar sind.

${ }^{8}$ Die gezeigten Klassenprofile wurden anhand einer sogenannten Maximum-Likelihood-Schätzung ermittelt. Mit andern Worten stellen sie jene Klasseneinteilung (der Grundgesamtheit) dar, die am wahrscheinlichsten zu der von uns beobachteten Stichprobe geführt hat. Zur Interpretation ein Beispiel: Ein Anteil von 0,6 (oder 60\%) der Mitglieder der ersten (latenten) Klasse fühlt sich hinsichtlich ihrer Meinung zur Frage von Geschwindigkeitsreduktionen in einer Minderheitsposition, während dieser Anteil in der zweiten latenten Klasse nur 0,2 beträgt

${ }_{9}^{9}$ Als Relativierung ist auch noch zu bemerken, daß das empfundene Minderheitsgefühl nicht in jeder Beziehung gleich stark ist.

${ }^{10}$ Dieses zweistufige Vorgehen, das mit einer Faktorenanalyse zweiter Ordnung vergleichbar ist, wurde hauptsächlich deshalb gewählt, weil sonst (bei einem einstufigen Vorgehen) die Meinungen zu den Luftreinhaltemaßnahmen ein übergroßes Gewicht bekommen hätten.

"Solche experimentelle Vorgehensweisen haben den Nachteil, daß sie die Komplexität und Kontextabhängigkeit der realen Handlungssituationen zu wenig berücksichtigen (können). Ebenso trägt der Spielcharakter des Experimentes nicht zu einem realitätsgetreuen Verhalten der Probanden bei. Eine umfassende nichtexperimentelle Prüfung der Bedeutung der Kommunikation und der kommunikativen Rationalisierung zur Überwindung unweltrelevanter Dilemmasituationen wurde bisher noch nicht versucht, wird gegenwärtig jedoch vom Autor dieses Beitrags vorbereitet.

\section{Literatur}

CLOGG, C. C., GOODMAN, L. A. (1984): Latent structure analysis of a set of multidimensional contingency tables. In: Journal of the American Statistical Association. Bd. 79, Nr. 388 , S. $762-711$. 
BRECHNER, K. C. (1977): An experimental analysis of social traps. In: Journal of Experimental Social Psychology. Bd. 13 S. $552-564$.

DAWES, R. M. (1980): Social Dilemmas. In: Annual Review of Psychology. Bd. 31, S. 169-193.

DAWES, R. M., McTAVISH, J., SHAKLEE, H. (1977): Behavior, communication, and assumptions about other people's behaviour in a common's dilemma situation. In: Journal of Personality and Social Psychology. Bd. 35, S. 1-11.

EDNEY, J. J., HARPER, C. (1978): The effects of information in a resource management problem: A social trap analog. In: Human Ecology. Bd. 6, S. 387-395.

ERNSTE, H., FISCHER, M. M. (1991): Latent class modelling and typological analysis. In: Sistemi Urbani. Bd. 13, No. 1-3, S. $163-174$

HABERMAS, J. (1987): Theorie des kommunikativen Handelns. Band 1: Handlungsrationalität und gesellschaftliche Rationalisierung. Band 2: Zur Kritik der funktionalistischen Vernunft. Suhrkamp, 4. Aufl., Frankfurt am Main.

HAGENAARS, J.A.P. (1990): Categorical Longitudinal Data: Log-Linear Panel, Trend, and Cohort Analysis. Sage, Newbury Park.

HARDIN, G. (1968): The Tragedy of the Commons. In: Science. Bd. 162, S. 1243-1248
KUNNEMAN, H. (1985): Habermas' Theorie van het communicatieve handelen. Een samenvatting. Boom, 3. Aufl. Meppel.

McCUTCHEON, A. L. (1987): Latent Class Analysis. Quantitative Applications in the Social Sciences. Bd. 64, Sage, Newbury Park.

MOSLER, H.J. (1990): Selbstorganisation von umweltgerechtem Handeln: Der Einfluß von Vertrauensbildung auf die Ressourcennutzung in einem Umweltspiel. Diss. Universität Zürich.

REESE-SCHÄFER, W. (1991): Jürgen Habermas. Campus, Frankfurt am Main.

REICHERT, D., ZIERHOFER, W. (unter Mitarbeit von Bättig, C. Ernste, H., Steiner, D., Vetterli M.) (1993): Umwelt zur Sprache bringen. Über das umweltverantwortliche Handeln, die Wahrnehmung der Waldsterbensdiskussion und den Umgang mit Unsicherheit. Westdeutscher Verlag, Opladen.

SEN, A. K. (1977): Rational Fools: a Critique of the Behavioral Foundations of Economic Theory. In: Philosophy and Public Affairs. Bd. 6, S. 317-344

SUDGEN, R. (1984): The Supply of Public Goods Through Voluntary Contributions. In: The Economic Journal. Bd. 94 S. $772-787$.

WEIMANN, J. (1991): Umweltökonomie. Eine theorieorientierte Einführung. Springer, 2. Aufl., Berlin. 